\title{
Graded-index fibers, Wigner-distribution functions, and the fractional Fourier transform
}

\author{
David Mendlovic, Haldun M. Ozaktas, and Adolf W. Lohmann
}

\begin{abstract}
Two definitions of a fractional Fourier transform have been proposed previously. One is based on the propagation of a wave field through a graded-index medium, and the other is based on rotating a function's Wigner distribution. It is shown that both definitions are equivalent. An important result of this equivalency is that the Wigner distribution of a wave field rotates as the wave field propagates through a quadratic graded-index medium. The relation with ray-optics phase space is discussed.

Key words: Fourier optics, optical information processing, fractional Fourier transforms, Wignerdistribution functions, graded-index media, spatial filtering.
\end{abstract}

\section{Introduction}

Recently, two distinct definitions of the fractional Fourier transform have been given. In the first one $^{1-3}$ the fractional Fourier transform was defined physically, based on propagation in quadratic gradedindex media (GRIN media). The ath fractional Fourier transform of a function was defined as follows: Let the original function be input from one side of a quadratic GRIN medium, at $z=0$. Then, the light distribution observed at the plane $z=z_{0}$ corresponds to the $a$ equal to the $\left(z_{0} / L\right)$ th fractional Fourier transform of the input fraction, where $L \equiv(\pi / 2)$ $\left(n_{1} / n_{2}\right)^{1 / 2}$ is a characteristic distance. The $a$ equal to the first Fourier transform, observed at $z_{0}=L$, corresponds to the ordinary Fourier transform, by design.

The second definition is based on the Wignerdistribution function ${ }^{4}$ (WDF). Here one calculates the fractional Fourier transform by finding the WDF of the input image, rotating it by an angle $\alpha=a \pi / 2$, and performing the inverse Wigner transform.

Both definitions fulfill two natural postulates: (i) The $a$ equal to the first Fourier transform corresponds to the ordinary Fourier transform. (ii) The fractional operator is additive, i.e., the $a$ th transform

D. Mendlovic is with the Faculty of Engineering, Tel-Aviv University, Tel-Aviv 69978, Israel. H. M. Ozaktas is with the Department of Electrical Engineering, Bilkent University, Bilkent, Ankara 06533, Turkey. A. W. Lohmann is with the Angewandte Optik, Erlangen University, Erlangen 8520, Germany.

Received 8 March 1993; revised manuscript received 22 November 1993.

0003-6935/94/266188-06\$06.00/0.

(c) 1994 Optical Society of America. of the $b$ th transform is equal to the $(a+b)$ th transform.

In this study we show that both definitions of the fractional Fourier transform are equivalent. The fact that two distinct definitions turn out to be identical supports the claim as to the naturalness and intrinsicalness of the definitions. To quote Minsky, "Proof of the equivalence of two or more definitions always has a compelling effect when the definitions arise from different experiences and motivations."

We also arrive at the result that the effect of propagation through GRIN media can be described as a rotation of the Wigner distribution of the input function. Thus this study answers two questions:

(1) How does the Wigner distribution of an input function change while propagating through a GRIN medium?

(2) Are the two fractional-Fourier-transform definitions fully equivalent?

In the following we show that the answer of the first question is simply that the Wigner distribution rotates uniformly with propagation distance. This answer leads to the conclusion that both fractionalFourier-transform definitions are equivalent. Section 2 gives some mathematical details about the WDF. Sections 3 and 4 describe the two fractionalFourier-transform definitions, and Section 5 gives the mathematical proof that both definitions are fully equivalent. Section 6 is a discussion and conclusion.

The analyses presented in this paper are for onedimensional (1-D) input functions, for notational convenience. However, all results can be extended trivially to higher dimensions, as is shown in Ref. 4 . 


\section{About the Wigner-Distribution Function}

The Wigner-distribution function ${ }^{6}$ (WDF) is a joint space-frequency (or time-frequency) representation of a signal. It describes the signal completely, displaying time and frequency information simultaneously. Applications of the WDF can be found in optics ${ }^{7,8}$ and in the representation of speech. ${ }^{9}$ The WDF of a 1-D signal $f(x)$ can be defined as

$$
W(x, \nu)=\int f\left(x+x^{\prime} / 2\right) f^{*}\left(x-x^{\prime} / 2\right) \exp \left(-2 \pi i \nu x^{\prime}\right) \mathrm{d} x^{\prime} .
$$

Using the conventional Fourier-transform operation,

$$
\tilde{f}(\nu)=\mathscr{F}\{f(x)\}=\int f(x) \exp (-2 \pi i v x) \mathrm{d} x,
$$

we can write an equivalent form of the WDF definition as

$$
W(x, v)=\int \tilde{f}\left(\nu+v^{\prime} / 2\right) \tilde{f}^{*}\left(\nu-v^{\prime} / 2\right) \exp \left(2 \pi i v^{\prime} x\right) \mathrm{d} v^{\prime}
$$

Properties of the WDF can be found in Refs. 4 and 8. Here we mention three of them. First is the effect of free-space propagation in the $z$ direction on the WDF. It was shown ${ }^{4}$ that such propagation causes the WDF to be sheared in the $x$ direction. The second is the effect of passage through a thin lens. This causes the WDF to be sheared in the $v$ direction. The third concerns the relation between the WDF of an object and the WDF of the conventional Fourier transform of the same object. From Eqs. (1) and Eq. (3) one can notice that we get a $\pi / 2$ rotation of the WDF. Another way to see this is to combine the effects of free-space propagation and passage through a thin lens in the form of a $2 f$ optical Fourier-transforming setup. First, we may perform the $x$ shearing associated with free-space propagation along a distance $f$, then the $v$ shearing associated with passage through a lens with focal length $f$, and finally another $x$ shearing associated with another free-space propagation along a distance $f$. The final result is a rotation by $\pi / 2$ of the original WDF. For a detailed discussion of these consecutive operations with illustrated examples, see Ref. 4.

\section{Fractional Fourier Transforms: Graded-Index Media Definition}

In this section the GRIN definition of the fractional Fourier transform is given. The reader can find more details in Refs. 1-3. The ath Fourier transform of a function $f(x)$ is denoted as $\mathscr{F}\{f(x)\}$. As was mentioned in the introduction, we require that our definition satisfy two basic postulates. First, $\mathscr{F}^{1} f$ should be the usual Fourier transform. Our second postulate requires that $\mathscr{F}^{a}\left\{\mathscr{F}^{b} f\right\}=\mathscr{F}^{a+b} f$.
Consistent with our two postulates, we suggested ${ }^{1-3}$ defining the fractional Fourier transform as the change of the field caused by propagation along a quadratic GRIN medium by a length proportional to $a$. Such a medium has a refractive-index profile given by ${ }^{10}$

$$
n^{2}(x)=n_{1}^{2}\left[1-\left(n_{2} / n_{1}\right) x^{2}\right],
$$

where $n_{1}$ and $n_{2}$ are the GRIN-medium parameters and $x$ is the 1-D coordinate. The eigenmodes of quadratic GRIN media are the Hermite-Gaussian (HG) functions, which form an orthogonal and complete basis set. The $m$ th member of this set is expressed as

$$
\Psi_{m}(x)=H_{m}\left(\frac{\sqrt{2} x}{\omega}\right) \exp \left(-\frac{x^{2}}{\omega^{2}}\right)
$$

where $H_{m}$ is a Hermite polynomial of order $m$ and $\omega$ is a constant that is connected with the GRIN-medium parameters. An extension to two lateral coordinates $x$ and $y$ is straightforward, with $\Psi_{m}(x) \Psi_{n}(y)$ as elementary functions. The lower-order $\mathrm{HG}$ polynomials are

$$
\begin{aligned}
& H_{0}(x)=1, \\
& H_{1}(x)=2 x, \\
& H_{2}(x)=4 x^{2}-2 .
\end{aligned}
$$

For a given wavelength $\lambda$ each HG mode propagates through the GRIN medium with a different group velocity and thus a different propagation constant,

$$
\begin{aligned}
\beta_{m} & =k\left[1-\frac{2}{k}\left(\frac{n_{2}}{n_{1}}\right)^{1 / 2}\left(m+\frac{1}{2}\right)\right]^{1 / 2} \\
& \approx k-\left(\frac{n_{2}}{n_{1}}\right)^{1 / 2}\left(m+\frac{1}{2}\right),
\end{aligned}
$$

with $k=2 \pi / \lambda$. Any function $f(x)$ can be expressed in terms of the HG basis set as

$$
f(x)=\sum_{m} A_{m} \Psi_{l m}(x)
$$

with

$$
A_{m}=\int_{-\infty}^{\infty} f(x) \Psi_{m}(x) / h_{m} \mathrm{~d} x
$$

where $h_{m}=2^{m} m ! \sqrt{\pi} \omega / \sqrt{2}$.

Now, the fractional Fourier transform of $f(x)$ of order $a$ is defined as

$$
\begin{aligned}
\mathscr{F} a\{f(x)\} & =\sum_{m} A_{m} \Psi_{m}(x) \exp \left(i \beta_{m} a L\right) \\
& \approx \sum_{m} A_{m} \Psi_{m}(x) \exp \left\{i\left[k-\left(\frac{n_{2}}{n_{1}}\right)^{1 / 2}\left(m+\frac{1}{2}\right) a L\right]\right\} .
\end{aligned}
$$


$L=(\pi / 2)\left(n_{1} / n_{2}\right)^{1 / 2}$ is the GRIN length that results in the conventional Fourier transform. It was shown ${ }^{1}$ that the above two postulates are satisfied by this definition. In the same reference we also discussed and proved some of the properties of fractional Fourier transforms, such as linearity, self-imaging parameters, and intensity shift variance/invariance (as with the common Fourier transform), and we generalized to complex values of the order $a$ (which can be physically realized by attenuating or amplifying media).

\section{Fractional Fourier Transforms:}

\section{Wigner-Distribution-Function Definition}

In Ref. 4 the fractional-Fourier-transform operation is defined as a rotation of the WDF by an angle $\alpha=$ $a \pi / 2$. It is shown in this reference that the two postulates of Section 3 are again fulfilled. It is relevant to point out that the WDF of a 1-D function is a two-dimensional function, and the rotation interpretation is clear. In Ref. 4 the same rotation strategy was generalized for two-dimensional signals, i.e., images whose WDF's are four-dimensional distributions. Since any rotation can be performed as three shearing operations $(x, v$, and $x$ shearings or $v, x$, and $v$ shearings), it was suggested ${ }^{4}$ that the system of Fig. 1 be used to perform the fractional Fourier transform with optical means. The first lens performs the first shearing, the propagation through free space is equivalent to the second shearing, and the second lens is for the third shearing. It was suggested that two parameters be used, $Q$ and $R$ (see Fig. 1), with

$$
f=f_{1} / Q, \quad z=f_{1} R,
$$

where $f_{1}$ is an arbitrary length, $f$ is the lenses' focal length, and $z$ is the distance between the lenses. For a fractional-Fourier-transform order, $a, Q$ and $R$ should be chosen as

$$
Q=\tan (\alpha / 2), \quad R=\sin (\alpha)
$$

with

$$
\alpha=a \pi / 2
$$

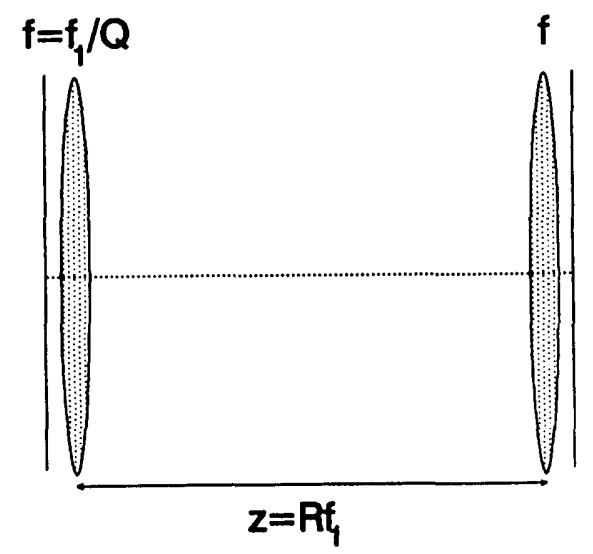

Fig. 1. Setup for performing a two-dimensional fractional Fourier transform according to the WDF definition.

\section{Equivalence Proof}

In this section we prove that both fractional-Fouriertransform definitions are equivalent. The strategy of the following proof is to calculate the action of the optical configuration of Fig. 1 and to show that it is equivalent to propagation through a GRIN medium of a certain length. In this section the operator $\mathscr{F}$ denotes the WDF-based fractional-Fourier-transform operator. Therefore for the WDF fractional-Fouriertransform definition, because at this moment we cannot be sure whether it is the same operation as $\mathscr{F}$, we rename it temporarily as $\mathscr{G}$ instead of $\mathscr{F}$, and the final result is that $\mathscr{G}=\mathscr{F}$.

According to the WDF fractional-Fourier-transform definition, by analyzing the optical configuration of Fig. 1, we can write

$$
\begin{aligned}
\mathscr{G}^{a}[f(x)]= & C_{1} \exp \left[-i \pi\left(Q-\frac{1}{R}\right) \frac{x_{0}^{2}}{\lambda f_{1}}\right] \int f\left(x_{0}\right) \\
& \times \exp \left[-i \pi\left(Q-\frac{1}{R}\right) \frac{x_{0}^{2}}{\lambda f_{1}}\right] \\
& \times \exp \left(-i 2 \pi \frac{x x_{0}}{\lambda f_{1} R}\right) \mathrm{d} x_{0} .
\end{aligned}
$$

$C_{1}$ is constant. $f\left(x_{0}\right)$ can be written as a superposition of the HG function set [see Eq. (8)]:

$$
f\left(x_{0}\right)=\sum_{n} A_{n} \Psi_{n}\left(x_{0}\right),
$$

where the $A_{n}$ are calculated by use of Eq. (9). Let us substitute Eq. (15) into Eq. (14) while using $T=Q-$ $1 / R$ :

$$
\begin{aligned}
\mathscr{G}^{a} f(x)= & C_{1} \exp \left(-i \pi T \frac{x^{2}}{\lambda f_{1}}\right) \int \sum_{n} A_{n} \Psi_{n}\left(x_{0}\right) \\
& \times \exp \left(-i \pi T \frac{x_{0}^{2}}{\lambda f_{1}}-i 2 \pi \frac{x x_{0}}{\lambda f_{1} R}\right) \mathrm{d} x_{0} \\
= & \sum_{n} A_{n} \mathscr{G}^{a} \Psi_{n}(x) .
\end{aligned}
$$

First, we calculate $\mathscr{G}^{a} \Psi_{n}(x)$ using mathematical induction. We start by hypothesizing that, consistent with the propagation of the eigenmodes of GRIN media,

$$
\mathscr{G}^{a} \Psi_{n}(x)=C_{2} \exp \left[i(n+1) \phi_{c}\right] \Psi_{n}(x) .
$$

In this expression, $C_{2}$ and $\phi_{c}$ are constants. This induction hypothesis is shown in hold for $n=0$ and $n=1$. Then is shown that its truth for $n-1$ and $n$ implies its truth for $n+1$. This ensures that it is true for all $n$. First, we check the validity of the hypothesis for $n=0$. The zero-order HG function is

$$
\Psi_{0}(x)=\exp \left(-\frac{x^{2}}{\omega^{2}}\right) .
$$


From Eq. (14) one gets

$$
\begin{aligned}
\mathscr{G}^{a} \Psi_{0}(\mathrm{x})= & C_{1} \exp \left(-i \pi T \frac{x^{2}}{\lambda f_{1}}\right) \\
& \times \int \exp \left(-\frac{x^{2}}{\omega^{2}}-i \pi T \frac{x_{0}^{2}}{\lambda f_{1}}-i 2 \pi \frac{x x_{0}}{\lambda f_{1} R}\right) \mathrm{d} x_{0} .
\end{aligned}
$$

Using the known relation ${ }^{11}$

$$
\int_{-\infty}^{\infty} \exp \left(-p^{2} x^{2} \pm q x\right) \mathrm{d} x=\frac{\sqrt{\pi}}{p} \exp \left(\frac{q^{2}}{4 p^{2}}\right),
$$

with

$$
p^{2}=\frac{1}{\omega^{2}}+\frac{i \pi T}{\lambda f_{1}}, \quad q=\frac{i 2 \pi x}{\lambda f_{1} R}
$$

we get

$$
\begin{aligned}
\mathscr{G}^{a} \Psi_{0}(x)= & C_{1} \exp \left(-i \pi T \frac{x^{2}}{\lambda f_{1}}\right) \\
& \times \exp \left[\left(-\frac{\pi^{2} x^{2}}{\lambda^{2} f_{1}^{2} R^{2}}\right)\left(\frac{1}{\frac{1}{\omega^{2}}+\frac{\pi^{2} T^{2}}{\lambda^{2} f_{1}^{2}}}\right)\left(\frac{1}{\omega^{2}}-i \frac{\pi T}{\lambda f_{1}}\right)\right] \\
& \times\left(\frac{\pi}{\frac{1}{\omega^{2}}+i \pi \frac{T}{\lambda f_{1}}}\right)^{1 / 2} .
\end{aligned}
$$

In order to adjust the last equation to the form of Eq. (17), we choose $\omega$ so as to satisfy

$$
\frac{\lambda^{2} f_{1}^{2} R^{2}}{\pi^{2} \omega^{4}}+T^{2} R^{2}=1
$$

Now Eq. (22) looks the same as

$$
\begin{aligned}
\mathscr{G}^{a} \Psi_{0}(x)= & C_{1} \exp \left(-i \pi T \frac{x^{2}}{\lambda f_{1}}+i \pi T \frac{x^{2}}{\lambda f_{1}}-\frac{x^{2}}{\omega^{2}}\right) \\
& \times\left(\frac{\pi}{\frac{1}{\omega^{2}}+i \pi \frac{T}{\lambda f_{1}}}\right)^{1 / 2} \\
= & C_{1}\left[\frac{\lambda^{2} f_{1}^{2}}{\pi}\left(\frac{1}{\omega^{2}}-i \pi \frac{T}{\lambda f_{1}}\right)\right]^{1 / 2} C_{1} \Psi_{0}(x) .
\end{aligned}
$$

One can notice that the field distribution of Eq. (24) is exactly the same as that of Eq. (18). As for the coefficients $C_{1}$ and $C_{2}$, we need not demonstrate their equality explicitly since this is required anyway by the conservation of energy. The important parameters are the argument of $C_{2}$ and $\phi_{c}$ appearing in Eq. (17).
We assume

$$
\begin{aligned}
\arg \left(C_{2}\right) & =-\arg \left[\left(\frac{\pi}{\frac{1}{\omega^{2}}+i \pi \frac{T}{\lambda f_{1}}}\right)^{1 / 2}\right]+\frac{\pi}{2}, \\
\phi_{c} & =\arg \left(\frac{\pi}{\frac{1}{\omega^{2}}+i \pi \frac{T}{\lambda f_{1}}}\right)-\frac{\pi}{2} .
\end{aligned}
$$

From Eqs. (17) and (24) one can notice that these choices agree for the special case of $n=0$.

Now for the $n=1$ HG order,

$$
\Psi_{1}(\mathrm{x})=\frac{2 \sqrt{2} x}{\omega} \exp \left(-\frac{x^{2}}{\omega}\right) .
$$

Using a relation similar to Eq. (20) we can prove that

$$
\mathscr{G}^{a} \Psi_{1}(x)=C_{2} \exp \left(i 2 \phi_{c}\right) \Psi_{1}(x),
$$

exactly following Eq. (17).

So far we have demonstrated that Eq. (17) holds for the $n=0$ and $n=1$ cases. Let us now assume that Eq. (17) holds for $n-1$ and prove it for $n+1$ :

$$
\mathscr{G}^{a} \Psi_{n+1}(x)=C_{2} \exp \left[i(n+2) \phi_{c}\right] \Psi_{n+1}(x),
$$

where

$$
\Psi_{n+1}(x)=H_{n+1}\left(\frac{\sqrt{2} x}{\omega}\right) \exp \left(-\frac{x_{0}^{2}}{\omega^{2}}\right) .
$$

Substituting the last equation into Eq. (14), one gets

$$
\begin{aligned}
\mathscr{G}^{a} \Psi_{n+1}(x)= & C_{1} \exp \left(-i \pi \frac{T x^{2}}{\lambda f_{1}}\right) \int H_{n+1}\left(\frac{\sqrt{2} x}{\omega}\right) \\
& \times \exp \left(-\frac{x_{0}^{2}}{\omega^{2}}-i \pi \frac{T x_{0}^{2}}{\lambda f_{1}}-i 2 \pi \frac{x x_{0}}{\lambda f_{1} R}\right) \mathrm{d} x_{0} .
\end{aligned}
$$

A known property of the Hermite polynomials is ${ }^{12}$

$$
H_{n+1}(x)=2 x H_{n}(x)-2 n H_{n-1}(x) .
$$

On the basis of this property we get

$$
\mathscr{G}^{a} \Psi_{n+1}(x)=G_{1}(x)+G_{2}(x),
$$

with

$$
\begin{aligned}
G_{1}(x)= & C_{1} \exp \left(-i \pi \frac{T x^{2}}{\lambda f_{1}}\right) \int 2 x H_{n}\left(\frac{\sqrt{2} x}{\omega}\right) \\
& \times \exp \left(-\frac{x_{0}^{2}}{\omega^{2}}-i \pi \frac{T x_{0}^{2}}{\lambda f_{1}}-i 2 \pi \frac{x x_{0}}{\lambda f_{1} R}\right) \mathrm{d} x_{0}, \\
G_{2}(x)= & C_{1} \exp \left(-i \pi \frac{T x^{2}}{\lambda f_{1}}\right) \int(-2 n) H_{n-1}\left(-\frac{\sqrt{2} x}{\omega}\right) \\
& \times \exp \left(-\frac{x_{0}^{2}}{\omega^{2}}-i \pi \frac{T x_{0}^{2}}{\lambda f_{1}}-i 2 \pi \frac{x x_{0}}{\lambda f_{1} R}\right) \mathrm{d} x_{0} .
\end{aligned}
$$


The induction assumption for $n-1$ leads to

$G_{1}(x)=C_{2}(-2 n) H_{n-1}\left(\frac{\sqrt{2} x}{\omega}\right) \exp \left(-\frac{x^{2}}{\omega^{2}}\right) \exp \left(i n \phi_{c}\right)$.

Now for $G_{2}(x)$, in Eq. (33) we can recognize a firstorder Fourier-transform integral; thus

$$
\begin{aligned}
G_{2}(x) & =C_{1} \exp \left(-i \pi \frac{T x^{2}}{\lambda f_{1}}\right) \\
& \times \mathscr{F}\left\{\frac{2 \sqrt{2}}{\omega} x H_{n}\left(\frac{\sqrt{2} x}{\omega}\right) \exp \left(-\frac{x_{0}^{2}}{\omega^{2}}-i \pi \frac{T x_{0}^{2}}{\lambda f_{1}}\right)\right\} .
\end{aligned}
$$

A well-known property of the conventional Fourier transform is

$$
\mathscr{F}\{x g(x)\}=\frac{1}{2 \pi} \frac{\mathrm{d}}{\mathrm{d} \nu} \mathscr{F}\{g(\mathrm{x})\} .
$$

With the relation $\nu=x /(\lambda f)$, the use of the Eq. (36), and the induction assumption for $n$, Eq. (35) becomes

$$
\begin{aligned}
G_{2}(x)= & \frac{2 \sqrt{2}}{\omega} \exp \left(-i \pi \frac{T x^{2}}{\lambda f_{1}}\right) \frac{\mathrm{d}}{\mathrm{d} x}\left\{C_{2} H_{n}\left(\frac{\sqrt{2} x}{\omega}\right) \exp \left(-\frac{x^{2}}{\omega^{2}}\right)\right. \\
& \left.\times \exp \left(i \pi \frac{T x^{2}}{\lambda f_{1}}\right) \exp \left[i(n+1) \phi_{c}\right]\right\} .
\end{aligned}
$$

Performing the differentiation $\mathrm{d} / \mathrm{d} x$ and using $^{13}$

$$
\frac{\mathrm{d}}{\mathrm{d} x} H_{n}(x)=2 n H_{n-1}(x)
$$

we get

$$
\begin{aligned}
G_{2}(x)= & \frac{2 \sqrt{2}}{\omega} i \frac{\lambda f_{1}}{2 \pi} \exp \left[i(n+1) \phi_{c}\right] C_{2} \\
& \times\left[2 x H_{2}\left(\frac{\sqrt{2} x}{\omega}\right) \exp \left(-\frac{x^{2}}{\omega^{2}}\right)\left(-\frac{1}{\omega^{2}}+i \frac{\pi T}{\lambda f_{1}}\right) \frac{\sqrt{2}}{\omega}\right. \\
& \left.\times 2 n H_{n-1}\left(\frac{\sqrt{2} x}{\omega}\right) \exp \left(-\frac{x^{2}}{\omega^{2}}\right)\right] .
\end{aligned}
$$

Now, Eq. (39) is substituted into Eq. (32). Using the explicit expression of $\phi_{c}$ [see Eq. (25)], we get

$$
\begin{aligned}
\mathscr{G}^{a} \Psi_{n+1}(x)= & \frac{2 \sqrt{2}}{\omega} i \frac{\lambda f_{1}}{2 \pi} \exp \left[i(n+1) \phi_{c}\right] C_{2} \exp \left(-\frac{x^{2}}{\omega}\right) \\
& \times\left[\frac{\pi}{i \lambda f_{1}} 2 x \exp i \phi_{c} H_{n}\left(\frac{\sqrt{2} x}{\omega}\right)\right. \\
& \left.+2 n H_{n-1}\left(\frac{\sqrt{2} x}{\omega}\right) \frac{\omega}{\sqrt{2}}\left(\frac{1}{\omega^{2}}-i \frac{\pi T}{\lambda f_{1}}\right)\right],
\end{aligned}
$$

or

$$
\begin{aligned}
\mathscr{G}^{a} \Psi_{n+1}(x)= & \exp \left[i(n+2) \phi_{c}\right] C_{2} \exp \left(-\frac{x^{2}}{\omega^{2}}\right) \\
& \times\left[2 x \frac{\sqrt{2}}{\omega} H_{n}\left(\frac{\sqrt{2} x}{\omega}\right)-2 n H_{n-1}\left(\frac{\sqrt{2} x}{\omega}\right)\right] .
\end{aligned}
$$

With Eq. (31) one gets

$$
\mathscr{G}^{a} \Psi_{n+1}(x)=C_{2} \exp \left[i(n+2) \phi_{C}\right] \Psi_{n+1}(x) .
$$

Since Eqs. (28) and (42) are exactly the same, the proof based on the induction technique is complete, and we can conclude that $\mathscr{G}=\mathscr{F}$.

So far we have derived the phase coefficient at the output of the system shown in Fig. 1 when a single HG order is presented as the input. The result is shown in Eq. (17). Let us now compare this result with the GRIN-media definition of the fractional Fourier transform. For an input function $f(x)$ the output of the configuration shown in Fig. 1 is

$$
\mathscr{F}^{a} f(x)=\sum_{n} A_{n} \exp \left[i(n+1) \phi_{c}\right] \Psi_{n}(x) .
$$

From Eq. (23) and the relation $T=Q-1 / R$ it can be proved that

$$
\exp \left(i \phi_{c}\right)=\cos (\phi)-i \sin (\phi)=\exp (-i \phi),
$$

where $\phi=a \pi / 2$. It can be seen that the GRIN fractional-Fourier-transform definition, Eq. (10), has exactly the same structure as Eq. (17) with

$$
\phi_{c}=-\left(\frac{n_{2}}{n_{1}}\right)^{1 / 2} a L=-a \frac{\pi}{2}=-\phi ;
$$

thus the two definitions are equivalent.

\section{Discussion and Conclusion}

In this section we highlight certain consequences of this equivalence. First, this implies that we have two equivalent ways of optically performing the fractional-Fourier-transform operation: we can use either a GRIN medium or the bulk system of Fig. 1. The GRIN-medium-based system can be considered to be more compact; however, available GRIN media have a limited space-bandwidth product.

Another implication of this equivalence is the fact that propagation through quadratic GRIN media results in a rotation of the Wigner-distribution function. This is because the bulk-optics system of Fig. 1 is fully equivalent to a GRIN medium of length $a L$. Until now, GRIN media have been handled mostly as ray-optics elements, mainly ${ }^{10}$ because of the lack of simple interpretations of its effect on the wave function of light passing through it. With the Wignerdistribution function we have a powerful tool for 
analyzing and designing systems with GRIN-media devices.

Additional insight into the rotation of the Wigner distribution can be gained by examination of the ray-optics analog of Wigner space, which is a particular type of phase space. We refer to the ray-optics analog of the Wigner space as the ray-optics phase space. Let a particular paraxial ray be characterized by its radial distance $r$ and slope $s$, both with respect to the optical axis at a particular axial position $z$. Then, the effect of passing through any optical system on this ray can be described by a movement in $r-s$ space, which is our ray-optics phase space. For instance, free-space propagation corresponds to a horizontal displacement, whereas focusing by a lens corresponds to a vertical displacement. This rayoptics phase space is analogous to Wigner space because of the correspondence between spatial frequency and the angle made with the optical axis.

Let us now consider a bundle of rays with a uniform spread of $r$ and $s$ (represented by a rectangular region in $\mathbf{r}-s$ space) and consider how this ray bundle is transformed as it passes through a quadratic GRIN medium. It is known that $r$ and $s$ obey the following equations in such media ${ }^{10}$ :

$$
\begin{aligned}
& r(z+\Delta z)=r(z) \cos (\pi \Delta z / 2 L)-s(z) \sin (\pi \Delta z / 2 L), \\
& s(z+\Delta z)=r(z) \sin (\pi \Delta z / 2 L)+s(z) \cos (\pi \Delta z / 2 L),
\end{aligned}
$$

from which we can conclude that the region representing any given bundle of rays in phase space is rotated uniformly as we go from $z=0$ to $z=L$. Thus, just as the Wigner distribution does, the ray-optics phasespace distribution also rotates uniformly upon propagation through GRIN media.

In conclusion, propagation through quadratic GRIN media corresponds to rotation of the phase-space distributions. We have shown that this is true for two specific phase-space representations: the Wigner representation and the ray-optics phase-space representation. ${ }^{1}$
We make a final remark about the applicability: the fractional Fourier transform is a generalization of the standard Fourier transform. The standard Fourier transform is heavily involved in optical applications, as the term Fourier optics implies. Hence it seems reasonable to expect optical applications when the new transform penetrates optics. Several directions are suggested in Ref. 2.

We dedicate this paper to the friendship of the German, Israeli, and Turkish peoples.

\section{References}

1. D. Mendlovic and H. M. Ozaktas, "Fractional Fourier transforms and their optical implementation: I," J. Opt. Soc. Am. A 10, 1875-1881 (1993).

2. H. M. Ozaktas and D. Mendlovic, "Fractional Fourier transforms and their optical implementation: II," J. Opt. Soc. Am. A 10, 2522-2531 (1993).

3. H. M. Ozaktas and D. Mendlovic, "Fourier transforms of fractional orders and their optical interpretation," Opt. Commun. 101, 163-169 (1993).

4. A. W. Lohmann, "Image rotation, Wigner rotation, and the fractional Fourier transform," J. Opt. Soc. Am. A 10, 21812186 (1993).

5. M. Minsky, Computation, Finite and Infinite Machines (Prentice-Hall, Englewood Cliffs, N.J., 1967), p. 111.

6. E. Wigner, "On the quantum correction for thermodynamics equilibrium," Phys. Rev. 40, 749-759 (1932).

7. M. J. Bastiaans, "Wigner distribution function and its application to first-order optics," J. Opt. Soc. Am. 69, 1710-1716 (1979); "The Wigner distribution function applied to optical signals and systems," Opt. Commun. 25, 26-30 (1978).

8. K. H. Brenner and A. W. Lohmann, "Wigner distribution function display of complex 1-D signals," Opt. Commun. 42, 310-314 (1982).

9. H. O. Bartelt, K. H. Brenner, and A. W. Lohmann, "The Wigner distribution function and its optical production," Opt. Commun. 32, 32-38 (1980).

10. A. Yariv, Optical Electronics, 3rd ed. (Holt, New York, 1985).

11. I. S. Gradshteyn and I. M. Ryzhik, Table of Integrals, Series and Products (Academic, New York, 1980).

12. B. E. A. Saleh and M. C. Teich, Fundamental of Photonics (Wiley, New York, 1991), p. 102, Eq. (3.3.5).

13. M. Abramovitz and I. A. Stegun, Handbook of Mathematical Functions (Dover, New York, 1970), Eq. (22.8.7). 